\title{
OPTIMAL CONFIGURATIONS FOR ROTATING SPACECRAFT FORMITIONS
}

\author{
Steven P. Hughes* and Christopher D. Hall ${ }^{\dagger}$
}

In this paper a new class of formations that maintain a constant shape as viewed from the Earth is introduced. An algorithm is developed to place $n$ spacecraft in a constant shape formation spaced equally in time using the classical orbital elements. To first order, the dimensions of the formation are shown to be simple functions of orbit eccentricity and inclination. The performance of the formation is investigated over a Keplerian orbit using a performance measure based on a weighted average of the angular separations between spacecraft in formation. Analytic approximations are developed that yield optimum configurations for different values of $n$. The analytic approximations are shown to be in excellent agreement with the exact solutions.

\section{INTRODUCTION}

Clusters of low-performance spacecraft flying in formation may provide enhanced performance over single high-performance spacecraft. This is especially true for remote sensing missions where interferometry or stereographic imaging may provide higher resolution data. There are many possibilities for configuring formations of satellites for such missions, but there are relatively few established performance measures for evaluating the effectiveness of particular configurations. This is especially important as configurations vary during an orbit due to orbital dynamics, and over longer time scales due to perturbations.

Significant attention has been given in the literature to many aspects of the distributed spacecraft concept. A preliminary feasibility study of formation flying technologies was performed by Folta et al. ${ }^{1}$ At the time of study it was determined that limitations in pointing accuracy could not meet an $80 \%$ overlap in the presence of errors. Further investigations were performed by Folta et al. ${ }^{2}$ to understand how to relate observations from two spacecraft, to determine the effects of perturbations. and to confirm previous results. The formation flying concept was revisited by Folta et $a l^{3}$ in 1996 and the feasibility of formation flying for particular missions in light of new advances in technology and autonomy was investigated. The dynamics of an

\footnotetext{
- Graduate Research Assistant; Currently, Aerospace Engineer, Guidance, Navigation, and and Control Center. N.ASA Goddard Space Flight Center, (301) 286-0145, (301) 286-0369 (FAX), email: shughes@pop500.gsfc.nasa.gov

${ }^{\dagger}$ Assistant Professor, Department of Aerospace and Orean Engineering, Virginia Tech., (540) 231-2314. (540) 231-3632 (FAX), email: challoae.vi edn
} 


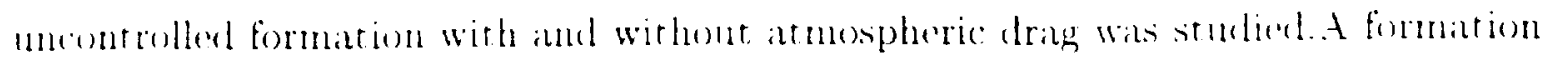

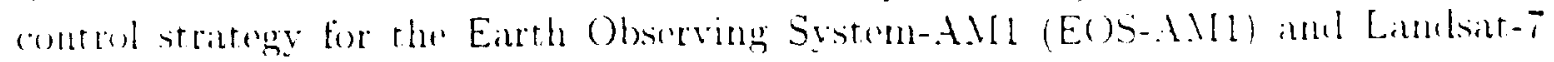

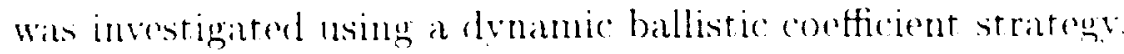

DeCout developed a three-satellite station-keeping strategy describing parths to perform very long baseline optical interferometry. DeCou concluded that the thrusting required is low enough to allow missions of up to ten vears using ion thrusters without refueling. Gramling et $a .^{5}$ demonstrated the possibility of using Goddard Space Flight Center's (GSFC) Onboard Navigation System (ONS) for relative navigation of the EO-1/Landsat- 7 spacecraft formation. Hartman et al. ${ }^{6}$ extended the study of GSFC's ONS performance for spacecraft formation flying. Specifically Hartman investigated orbit determination and prediction accuracies and their impact on the relative separation errors.

The use of GPS for relative navigation of formation flying spacecraft has been studied by Guinn et $a l^{7}$ and for both relative position and attitude sensing by Adams et $a l^{8}$ and How et al. ${ }^{9}$ Adams et $a l^{8}$ presented results from a GPS-based relative navigation and attitude sensing system developed in the laboratory.

In this paper, we introduce a class of formations we call rotating formations, where the configuration remains relatively constant during an orbit. A detailed analysis of the performance of this class of formations, including an approximate analytical determination of the optimal configuration based on an angular performance measure is presented. The new class of formations involves an elliptical arrangement of satellites in slightly elliptical orbits, each inclined slightly with respect to the orbital plane of the reference circular orbit. Each orbit has the same semimajor axis, inclination with respect to the reference plane, eccentricity, and argument of periapsis. The position of each satellite is determined by these common elements, and by unique values for the right ascension of the ascending node and true anomaly at epoch. A general expression for these angles is developed in terms of the number of satellites in the formation, $n$, based on a equal-time distribution of the satellites. The relative configuration remains nearly constant during an orbit, as the satellites rotate about the reference circular orbit. In our development, the eccentricity $e$ and inclination $i$ are treated as design variables, and values are chosen to optimize an appropriate performance measure.

One measure of the performance of such a formation is the angular separation between the satellites, as this defines the performance of interferometry and of stereographic imaging. A particular instrument's effectiveness in these missions can be related to the angular separation, $\alpha_{i j}$, between the $i_{t h}$ and $j_{t h}$ satellites in the formation. For a specific value, $\alpha_{m}$, the performance of any pair of satellites will be maximized. Since the angular separation varies during the orbit, the performance measure is actually an orbit-averaged quantity depending on all the angular separations. For the rotating configuration developed here, the integrand of this performance measure is 
nearly enustant. especially for latege n, since the time it takes the formation an reath a meall cepulablent contiguration is $T / n$, where $T$ is the orbital periond. Thus the iutegrand rat be used as a proxy performance moasure. which allows the andytical derelopment of the measure with all orbital elements inchaled in the expression.

The full analytical expression of the performance measure is extremely complicated, and its complexity increases geometrically with $n$. We develop a relationship between the eccentricity and inclination that permits us to develop a series approximation for the performance measure. Using only terms to second order, simple approximations are developed for the values of $e$ and $i$ that maximize the formation performance. These approximations take the form of a function that depends only on $n$ multiplied by the optimal separation angle $\alpha_{m}$. Thus the optimal $e$ and $i$ can easily be computed for the different optimal separation angles associated with different instruments. The approximations exhibit excellent agreement with the exact solutions obtained using the orbit-averaged "exact" version of the performance measure. Furthermore, the functional dependence of the optimal $e$ and $i$ on the number of satellites, $n$, is recaptured by investigating the solutions for different values of $n$.

\section{DEVELOPMENT OF THE ROTATING FORMATION}

In this section, a formation of $n$ spacecraft is developed such that the satellites move along an ellipse-like shape in a relative reference frame. A configuration of $n$ spacecraft on an ellipse-like path is shown in Figure 1. In general, the horizontal dimension is defined as the longitudinal separation and the vertical separation as the latitudinal separation. The dimensions can be physically represented using the angular or spatial separations. To distinguish between the two representations, the angular dimensions of the ellipse are measured using the angles $\delta_{\text {lon }}$ and $\delta_{\text {lat }}$ to specify the longitudinal angular separation and the latitudinal separation respectively. When the dimensions of the ellipse are discussed in units of distance, the horizontal dimension is termed $d_{l o n}$ and the vertical dimension is called $d_{\text {lat }}$.

In the development of the rotating formation two coordinate systems are used: an inertial frame $\mathcal{F}_{R}$, and a relative reference frame $\mathcal{F}_{\text {rel }}$ defined by a reference circular orbit. The reference circular orbit is chosen to be equatorial with a true longitude at epoch of $\ell_{\text {ref }}$. The origin of $\mathcal{F}_{\text {rel }}$ is attached to a reference spacecraft that moves along the reference orbit. The $x_{r e l}$-axis is in the direction of the reference spacecraft's position vector. The $y_{r e l}$-axis lies along the reference spacecraft's velocity vector direction. By this definition the $y_{r e l}$-axis is always tangent to the reference orbit. The $z_{r e l}$-axis is chosen to complete the right-handed set, and is in the orbit normal direction.

Frame $\mathcal{F}_{R}$ is an inertial frame but it is not equivalent to the Earth-centered inertial frame $\mathcal{F}_{1}$ commonly found in the literature. The frame is fixed in inertial space with its origin at the center of the Earth. However, its axes can be defined by any arbitrary 


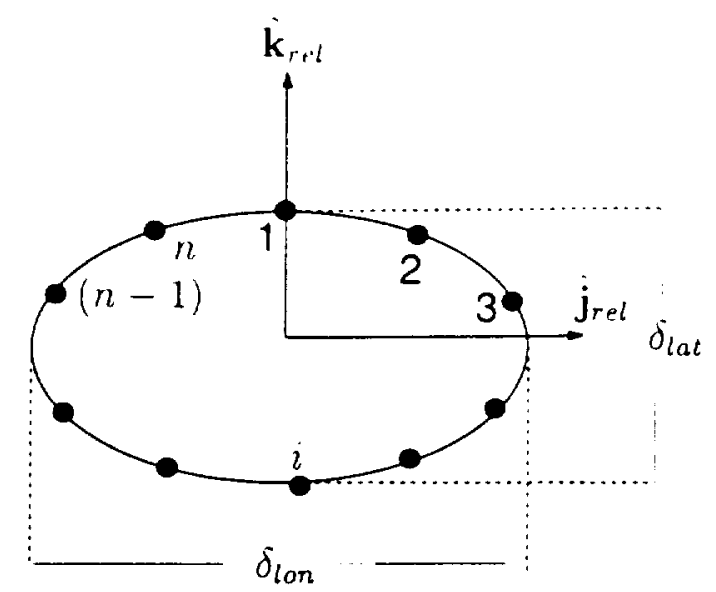

Figure 1: Geometry of the Rotating Formation

right-handed system. The result is that the $x_{R}-y_{R}$ plane of $\mathcal{F}_{R}$ is not the Earth's equatorial plane, it is the orbital plane of the reference circular orbit. The orbital elements used to describe the rotating formation are used according to their classical definition, but for generality they are measured with respect to $\mathcal{F}_{R}$ unless otherwise stated.

We wish to determine the dimensions of the relative orbit in terms of the orbital elements of the spacecraft in formation. To simplify the analysis the discussion is limited to orbits that are nearly circular and result in small displacements from the reference orbit. In doing so, we require that the spacecraft motion occurs in nearly the same plane as the reference motion. Therefore, the along-track displacements in the relative motion are primarily due to the eccentricity of the orbits. With these assumptions the dependence of $\delta_{l o n}$ and $\delta_{l a t}$ on the orbital elements can be determined.

A view of the ellipse-like path of the rotating formation in $\mathcal{F}_{R}$ is shown in Figure 2. The vector $\mathbf{r}_{1}$ describes the position of an arbitrary spacecraft. The true anomaly, $\nu$, for the spacecraft is measured from the $\hat{\mathbf{i}}_{1}$ axis. The quantity $y_{l o n}$ is the along-track component of angular separation of the spacecraft in $\mathcal{F}_{\text {rel }}$; i.e., $y_{\text {lon }}$ is defined as an angle and not a distance. From inspection of Figure 2, we see that the angle $\delta_{\text {lat }}$ is due to the difference in inclination between the orbit and the reference orbit. The expression for $\delta_{l a t}$ in terms of the inclination is simply

$$
\delta_{l a t}=2 i
$$

Recall that it is assumed that the out-of-plane motion does not affect the alongtrack angular separation. Therefore, an expression for $\delta_{\text {lon }}$ can be determined by investigating the projection of the motion in the $x_{R}-y_{R}$ plane as seen in Figure 3 . First an expression for $y_{l o n}$ is developed. From inspection of Figure 3. ylon can be written

$$
y_{l o n}=\nu-\theta
$$




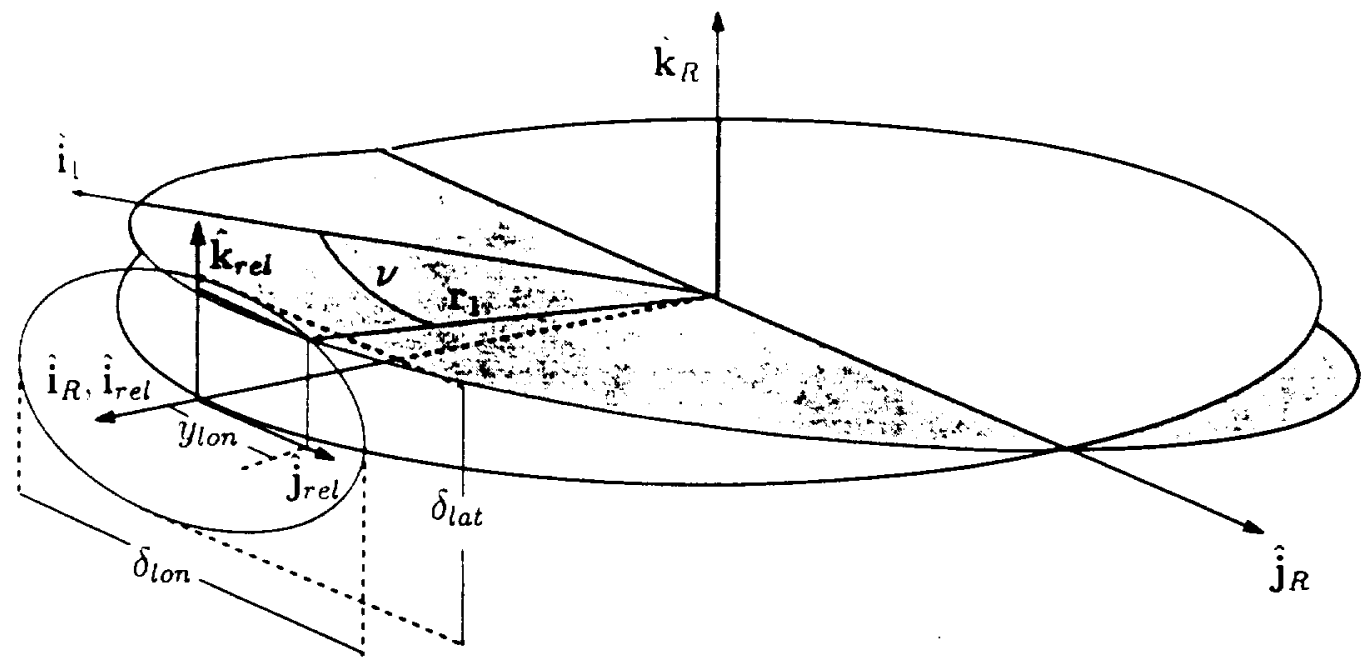

Figure 2: The Rotating Formation Seen in $\mathcal{F}_{R}$ and $\mathcal{F}_{\text {rel }}$

where $\theta$ is the angle between $\hat{\mathbf{i}}_{\text {rel }}$ and $\hat{\mathbf{i}}_{1}$. An expression for $\theta$ can be written as

$$
\theta=M_{0_{r e l}}+\eta t
$$

where $M_{0_{\text {rel }}}$ is the angle between $\hat{\mathbf{i}}_{\text {rel }}$ and $\hat{\mathbf{i}}_{1}$ at the initial epoch. The horizontal dimension, $\delta_{l o n}$, is simply twice the maximum in the along-track separation:

$$
\delta_{l o n}=\left.2 y_{l o n}\right|_{\max }
$$

For small $e$ we can write

$$
\nu=M_{0_{1}}+\eta t+2 e \sin \left(M_{0_{1}}+\eta t\right)
$$

as found in Battin, ${ }^{10}$ where $M_{0_{1}}$ is the mean anomaly of $\mathbf{r}_{1}$ at the initial epoch, and $\eta$ is the mean motion. Substituting Eqs. (3) and (5) into Eq. (2) we obtain

$$
y_{\text {lon }}=2 e \sin \left(M_{0_{1}}+\eta t\right)+M_{0_{1}}-M_{0_{\text {rel }}}
$$

Recall that $M_{0_{1}}$ and $M_{0_{\text {rel }}}$ are constants so

$$
\left.y_{\text {ton }}\right|_{\max }=2 e
$$

Substituting Eq. (7) into Eq. (4) we obtain

$$
\delta_{l o n}=4 e
$$

A similar result was arrived at by Chichka ${ }^{11}$ using a second-order approximation.

Equations (1) and (8) show the relationship between the dimension of the relative motion in terms of $e$ and $i$. However. we need to develop an algorithm to place $n$ spacecraft in an ellipse-like formation defined by $\delta_{l o n}$ and $\delta_{\text {lat }}$. 


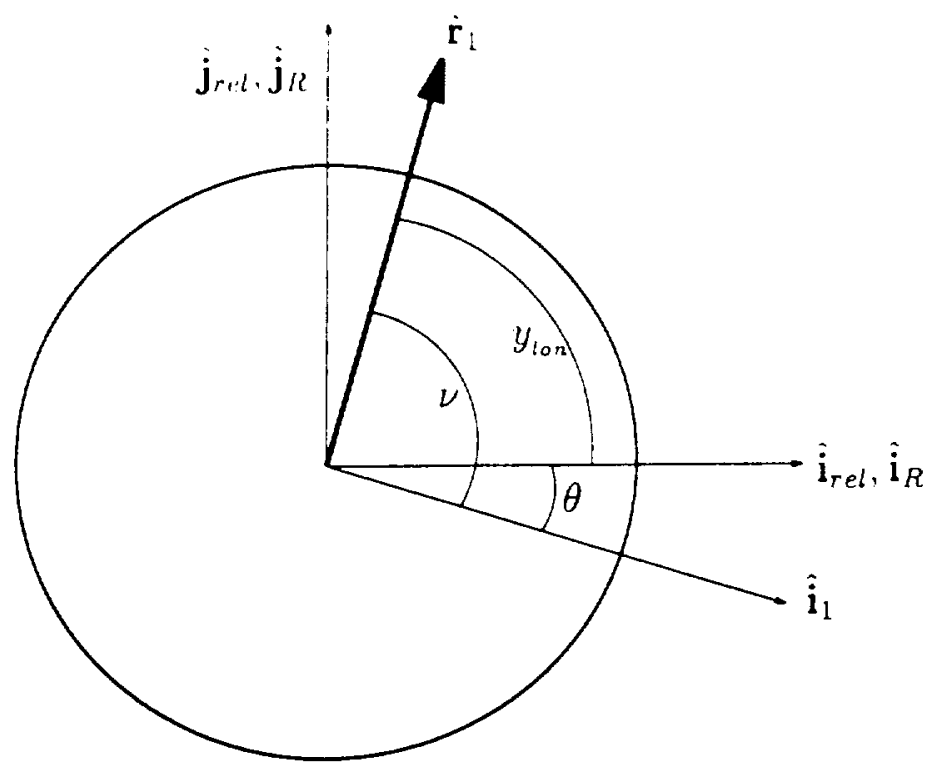

Figure 3: The Rotating Formation Projected into the $x_{R}-y_{R}$ Plane

\section{SPACECRAFT PLACEMENT IN THE ROTATING FORMATION}

In this section an algorithm to place $n$ spacecraft along the ellipse-like path in the relative reference frame is developed in terms of $\delta_{\text {lon }}$ and $\delta_{\text {lat }}$. The spacecraft can be placed in the formation in three different configurations: equal angle separations, equal arc length separations, and equal time separations. In this paper an algorithm is developed to place the spacecraft around the ellipse-like path with equal time separations. For circular formations, equal time separation is equivalent to equal arc length and equal angle separation. For ellipse-like formations the relationship between the three configurations is non-trivial. The formation is developed by first investigating the requirements to place a single spacecraft on an ellipse-like path defined by $\delta_{l o n}$ and $\delta_{l a t}$. Then, the method is generalized to include $n$ spacecraft spaced equally in time.

We know from Eqs. (1) and (8) that the dimensions of the relative motion path in $\mathcal{F}_{\text {rel }}$ depends only on $e$ and $i$. Therefore, for all the spacecraft to move along an ellipse of the same dimensions in $\mathcal{F}_{\text {rel }}$, all the orbits in the formation must have a common $e$ and $i$. Solving for $i$ in Eq. (1) yields

$$
i=\delta_{\text {lat }} / 2
$$

From Eq. (8) the eccentricity can be written as

$$
e=\delta_{l o n} / 4
$$

To ensure a cohesive formation, all the orbits must also have the same semimajor axis. Therefore, only the argument of periapsis. $\omega$. the longitude of ascending node, $\Omega$, and the true anomaly, $\nu$ may vary for the orbits in the rotating formation. 


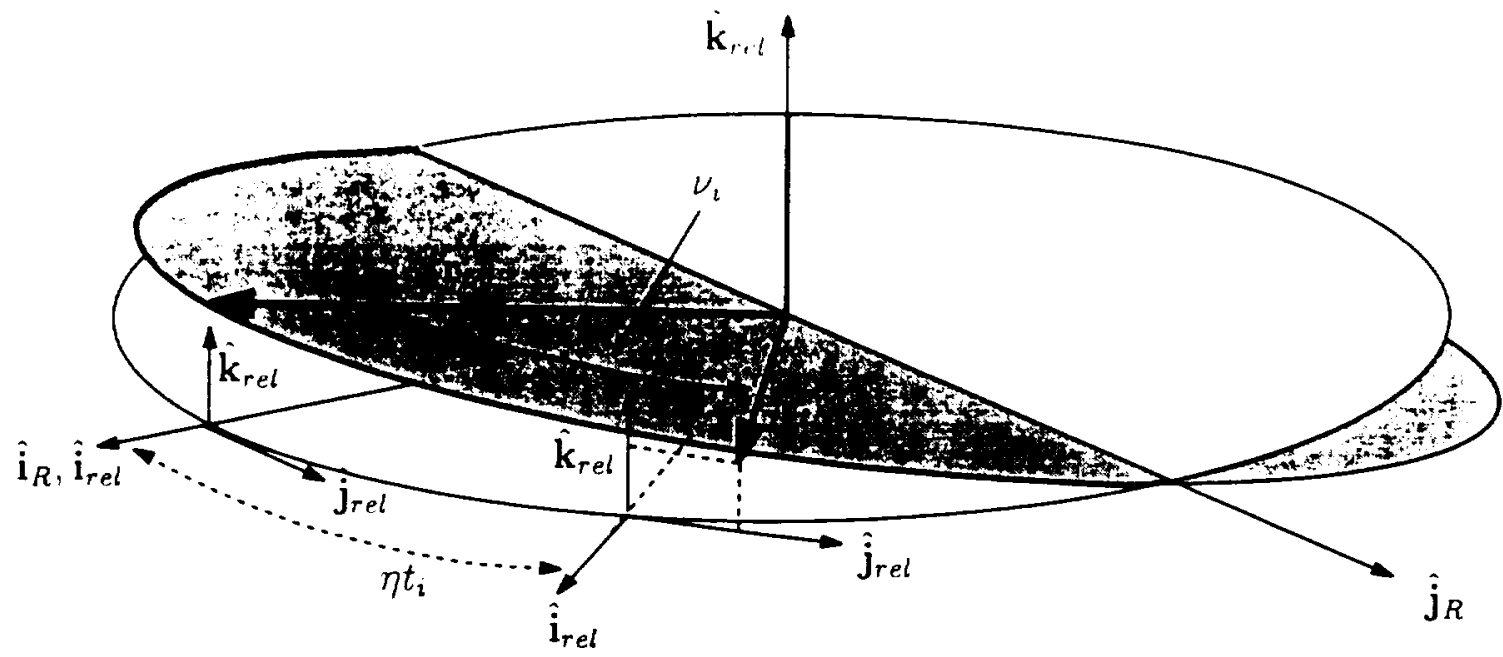

Figure 4: Time Evolution of the Rotating Formation

In Figure 4, the position of an arbitrary satellite at two different epochs is shown. At the initial epoch the position is described by $\mathbf{r}_{t_{0}}$; at a later epoch the position is described by $\mathbf{r}_{t_{i}}$. The origin of the reference orbital frame is chosen to lie along the $x_{R}$-axis at the initial epoch in a circular orbit whose plane of motion is the $x_{R}-y_{R}$ plane. We begin constructing the formation by placing a spacecraft in position 1 shown in Figure 1. The relative motion is chosen to be clockwise. For clockwise motion to occur the spacecraft at position 1 must be moving in the $y_{\text {rel }}$ direction faster than the origin of the reference orbit. Therefore, spacecraft 1 is positioned at the periapsis of its orbit:

$$
\nu_{1}=0
$$

The angles $\omega_{1}$ and $\Omega_{1}$ must be chosen to position the periapsis of orbit 1 over $\hat{\mathbf{i}}_{R}$. By inspection of Figure 4 we see that this is accomplished by choosing

$$
\Omega_{1}=3 \pi / 2 \quad \omega_{1}=\pi / 2
$$

In summary, the orbital elements to position a spacecraft at position 1 in $\mathcal{F}_{\text {rel }}$ at the initial epoch are

$$
a_{1}=a \quad e_{1}=\delta_{l o n} / 4 \quad i_{1}=\delta_{\text {lat }} / 2 \quad \omega_{1}=\pi / 2 \quad \Omega_{1}=3 \pi / 2 \quad \nu_{1}=0
$$

We now wish to generalize the algorithm to place $n$ satellites spaced equally in time along the ellipse-like path seen in Figure 1. For all satellites to move along the same path in $\mathcal{F}_{R}$, all the orbits must share a common $a, e$, and $i$. However $\omega, \Omega$, and $\nu$ must be determined for the remaining orbits. 
The time of separation, t, between spacecraft in an equal time formation is given ly

$$
t_{\mathrm{s}}=T / n=\frac{2 \pi}{n} \sqrt{\frac{0^{3}}{\mu}}
$$

To determine the orbital elements of the $i^{\text {th }}$ spacecraft in formation orbit 1 is propogated through time $t_{\imath}$ where

$$
t_{i}=(i-1) t_{s}
$$

The configuration of spacecraft 1 and the reference orbit at the initial epoch, $t_{0}$. and at $t_{i}$ can be seen in Figure 4 . We need to determine $\nu_{t_{1}}$, the value of $\nu$ for orbit 1 after the passage of time $t_{i}$. For small $e$ a first order expansion for $\nu$ is

$$
\nu_{t_{\mathrm{i}}}=M_{t_{\mathrm{i}}}+2 e \sin M_{t_{\mathrm{i}}}
$$

where

$$
M_{t_{\mathrm{i}}}=M_{0}+(i-1) \eta t_{s}
$$

Recall that the true anomaly for orbit 1 at the initial epoch is zero. Therefore, the mean anomaly of orbit 1 at the initial epoch, $M_{0}$, is also zero. Substituting Eq. (14) and (17) into Eq. (16) the relation for the true anomaly is obtained:

$$
\nu_{t_{i}}=\frac{2 \pi}{n}(i-1)+2 e \sin \left(\frac{2 \pi}{n}(i-1)\right)
$$

For a Keplerian orbit only $\nu$ changes with time. Therefore, the orbital elements to space $n$ spacecraft equally in time in $\mathcal{F}_{R}$ are known. However, we are interested in the elements that yield $n$ spacecraft spaced equally in time in $\mathcal{F}_{\text {rel }}$. Therefore, we need to determine the new configuration of $\mathcal{F}_{\text {rel }}$ after the passage of time $t_{i}$. Then the orbital elements to space $n$ spacecraft equally in time in $\mathcal{F}_{\text {rel }}$ can be determined by a coordinate transformation.

The frame $\mathcal{F}_{\text {rel }}$ is defined by a circular, equatorial reference orbit in $\mathcal{F}_{R}$. Therefore, the motion of the coordinate system $\mathcal{F}_{\text {rel }}$ after time $t_{i}$ is simply a 3-rotation through the angle $\theta=\eta t_{i}$. Performing a 3-rotation through $\eta t_{i}$ to $\mathcal{F}_{r e l}$ is equivalent to rotating $\Omega_{i}$ through an angle $\theta=-\eta t_{i}$. Therefore, to have $n$ spacecraft spaced equally in time in $\mathcal{F}_{\text {rel }}$ the node is written

$$
\Omega_{i}=3 \pi / 2-\eta t_{i}=\frac{3 \pi}{2}-\frac{2 \pi(i-1)}{n}
$$

In summary, to obtain the orbital elements to place the $i^{\text {th }}$ spacecraft in an equal time, rotating formation in $\mathcal{F}_{\text {rel }}$ orbit 1 is propogated through $t_{i}$ as defined in Eq. (15). Then the longitude of the ascending node is rotated backwards through $\eta t_{2}$ to $\Omega_{2}$ as defined by Eq. (19) to account for the motion of $\mathcal{F}_{\text {rel }}$. Hence $a, e, i$, and $\omega$ are the same for all orbits in the formation. The true anomaly and the longitude of ascending 
note are given by Ess. (L8) and (19). In general. the th set ot orbital elements is given by

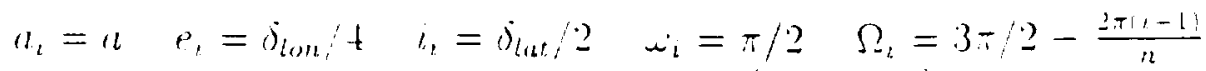

$$
\begin{aligned}
& \nu_{\imath}=\frac{2 \pi}{n}(i-1)+2 e_{i} \sin \left(\frac{2 \pi}{n}(i-1)\right)
\end{aligned}
$$

where $1 \leq i \leq n$.

\section{PERFORMANCE OF THE ROTATING FORMATION}

We wish to investigate the performance of the rotating formation using an appropriate metric. We propose that the performance of the formation can be evaluated by investigating the angular separations between spacecraft over the course of an orbit. $^{12}$ Specifically, an orbit-averaged quantity based on the sum of the instantaneous separation angles is considered:

$$
W_{\alpha}=\frac{1}{n_{s} T} \int_{0}^{T} \sum_{i=1}^{n-1} \sum_{j=i+1}^{n} w_{\alpha}\left(\alpha_{i j}\right) d t
$$

where $w_{\alpha}$ is an instantaneous position metric to be chosen by the analyst, $\alpha_{i j}$ is the angular separation between the $i^{\text {th }}$ and $j^{\text {th }}$ spacecraft, and $\eta_{s}$ is the number of unique separation angles given by

$$
n_{s}=\frac{n^{2}-n}{2}
$$

A measure of this form allows the analyst to define an instantaneous metric for the angular separation between a pair of spacecraft. The performance of all pairs in formation is evaluated accordingly and summed together to obtain a measure of the formation performance at a particular instance in time. By integrating the instantaneous metric over an orbit a measure of orbit effectiveness is obtained. A parabolic form is chosen for the instantaneous weight function, $w_{\alpha}$. In doing so, the flexibility is enabled to choose an upper and lower limit for the acceptable angular separations between two spacecraft as well as the optimum configuration for two spacecraft.

To solve the performance problem, the position information for all spacecraft in formation over an orbit period is needed. In general, spacecraft are subject to forces from the spherical primary as well as perturbations from atmospheric drag, solar radiation pressure, third body forces, and non-spherical effects. However, approximating the orbital dynamics with Keplerian motion is suitable for a preliminary analysis. Therefore, to obtain the conditions that yield an optimum configuration Eq. (21) must be solved for an optimum where the dynamics of the formation are described by the two body equation of motion with the initial conditions given by the orbital elements in $\mathrm{Eq}$. (20).

We now investigate optimum configurations of the formation according to the angular separation performance metric. 


\section{An Analytic Solution}

We wish to determine optimal configurations of the rotating formation in rems of the orbital elements defining the rotating formation. Hence, we seek the orbital elements that yield an optimum in $\mathrm{Eq}$. (21). In general. $W_{\alpha}$ does not permit a closedform analytic solution. However, some simplifying assumptions are made that allow the application of the metric to the rotating formation to obtain analytic results. In summary, a closed-form solution for the maximum in Eq. (21) is desired where

$$
w_{\alpha}\left(\alpha_{i j}\right)=c_{1} \alpha_{i j}^{2}+c_{2} \alpha_{i j}+c_{3}
$$

The performance measure seen in Eq. (21) is based on the angular separations between spacecraft, which are determined by the orbital elements in Eq. (20). For the rotating formation, the orbital elements are simple functions of $a, n, \delta_{l o n}$, and $\delta_{l a t}$. However, the angular separation between spacecraft does not change with semimajor axis. Therefore, the functional expression for $\alpha_{i j}$ is

$$
\alpha_{i j}=f\left(n, \delta_{l o n}, \delta_{l a t}\right)
$$

The parabolic instantaneous weight function, $w_{\alpha}$, can be uniquely defined by three constants $c_{1}, c_{2}$, and $c_{3}$. The result is that the performance problem has six design variables such that

$$
W_{\alpha}=f\left(n, \delta_{\text {lon }}, \delta_{\text {lat }}, c_{1}, c_{2}, c_{3}\right)
$$

The dimensions $\delta_{\text {lon }}$ and $\delta_{\text {lat }}$ can be written in terms of inclination and eccentricity therefore, $\alpha_{i j}$ can be expressed in terms of $n, e$, and $i$. A solution for $\alpha_{i j}$ in terms of $n, e$, and $i$ allows a solution for $W_{\alpha}$ of the form

$$
W_{\alpha}=f\left(n, e, i, c_{1}, c_{2}, c_{3}\right)
$$

An expression for $\alpha_{i j}$ can be obtained using the definition of the cross product:

$$
\left\|\mathbf{r}_{i} \times \mathbf{r}_{j}\right\|=r_{i} r_{j} \sin \alpha_{i j}
$$

For close formations $\alpha_{i j}$ is small so we can assume

$$
\sin \alpha_{i j} \approx \alpha_{i j}
$$

Solving for $\alpha_{i j}$ we obtain the expression

$$
\alpha_{i j}=\frac{\left\|\mathbf{r}_{\imath} \times \mathbf{r}_{j}\right\|}{r_{\imath} r_{j}}
$$

The vector $\mathbf{r}_{i}$ in the perifocal system can be expressed as

$$
\mathbf{r}_{i_{p}}=\frac{a\left(1-e^{2}\right)}{1+e \cos \nu_{i}}\left[\begin{array}{lll}
\cos \nu_{i} & \sin \nu_{i} & 0
\end{array}\right]^{T}
$$




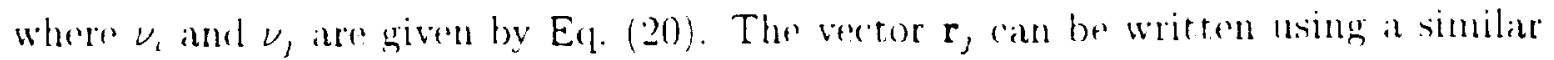
expresionn by replacing the subscript $i$ in $E$ (1. (30) with $J$.

T1) use $E_{(1 .}$ (29) the vectors must be rotated into a common reference framo using the appropriate rotation matrix. The rotation matrix to go from the perifocal system to $\mathcal{F}_{R}$ is constructed for the $i^{\text {th }}$ position vector using

$$
\mathbf{R}_{\mathbf{i}}^{I p}=\mathbf{R}_{3}\left(-\Omega_{i}\right) \mathbf{R}_{1}(-i) \mathbf{R}_{3}(-\omega)
$$

where $\Omega_{i}$ is given in the orbital element set in Eq. (20). Then the inertial representations of $\mathbf{r}_{i}$ and $\mathbf{r}_{j}$ can be written using

$$
\mathbf{r}_{i_{l}}=\mathbf{R}_{\imath}^{I p} \mathbf{r}_{i_{p}} \quad \mathbf{r}_{j I}=\mathbf{R}_{j}^{I p} \mathbf{r}_{j_{p}}
$$

Using the preceding, we have the information required to evaluate $\alpha_{i j}$ from Eq. (29). The expression, which is not shown here, is a non-trivial transcendental function of the form

$$
\alpha_{i j}=f(n, e, i)
$$

We now make some assumptions to arrive at a simpler form for $W_{\alpha}$.

\section{Simplifying Assumptions}

In general, solving $W_{\alpha}$ requires integrating the motion over an orbit. However, for special configurations of the rotating formation, the relative spacing between the spacecraft is nearly constant over an orbit. This is true for near circular rotating formations when

$$
\delta_{l o n} \approx \delta_{l a t}
$$

The relative positions are also nearly constant over an orbit for formations composed of a large number of spacecraft. In Figure 5, a rotating formation of four spacecraft is shown. The shaded circles represent the positions of four spacecraft at the initial epoch. The white circles represent the positions of the spacecraft after a small passage of time, $\Delta t$. The relative positions, and hence the angular separations, have changed after the time $\Delta t$. However, when the spacecraft at position 1 reaches position 2 the relative positions of the spacecraft will be nearly the same as at the initial epoch. The relative positions are not exactly the same because all of the spacecraft in formation are at different points in elliptical orbits. For example, for this configuration spacecraft 2 is at periapsis and spacecraft 1 is at a true anomaly given by Eq. (20) with $i=4$. Therefore, the time for spacecraft 1 to reach position 2 is slightly different from the amount of time for spacecraft 2 to reach position 3. For a four-spacecraft formation the relative positions are nearly the same after a passage of time $T / 4$. In general, for a formation of $n$ spacecraft. the relative positions are nearly 


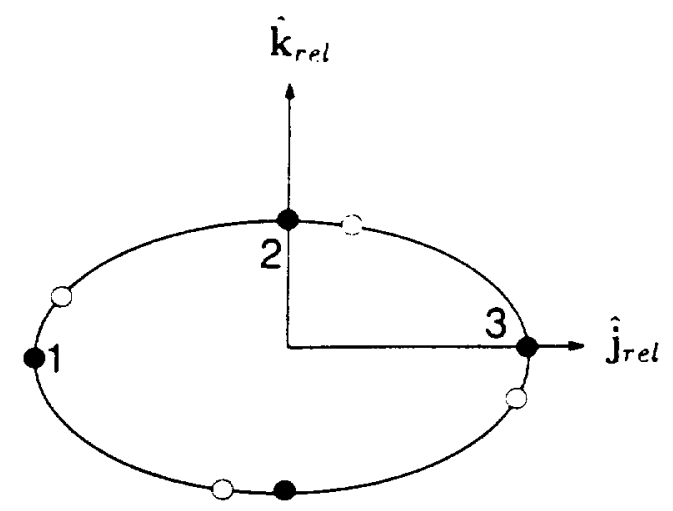

Figure 5: Rotating Formation of Four Spacecraft

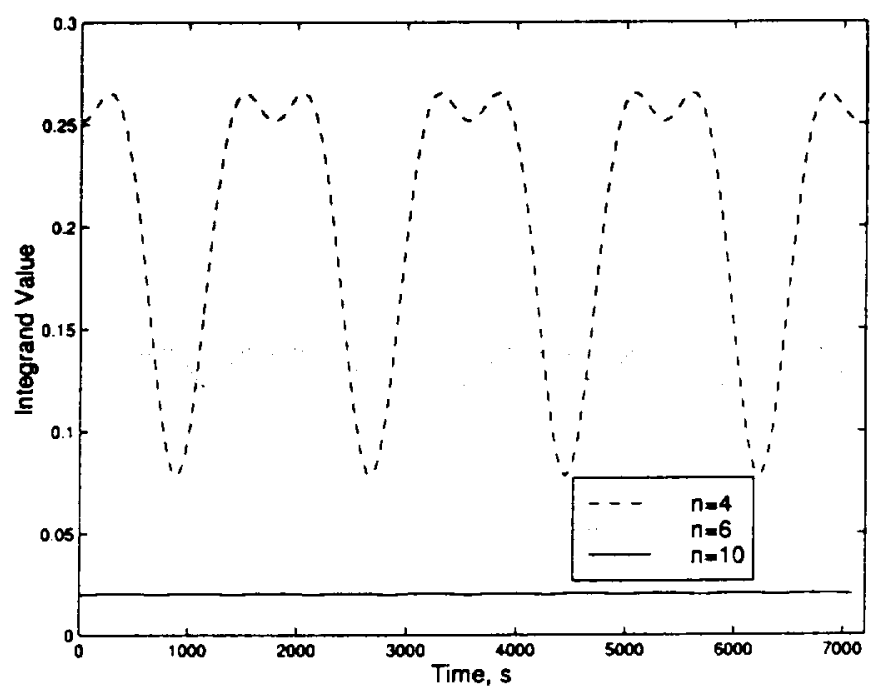

Figure 6: Integrand vs. Time for a Highly Elliptical Formation

equivalent after time $T / n$. Therefore, the period of the formation decreases with $n$. More importantly though, as $n$ increases the amplitude of the integrand in Eq. (21) goes to zero. This is demonstrated in Figure 6. A plot of the integrand over an orbit period for $n=4,6,10$ is shown for a highly elliptical formation with $d_{l o n}=6000 \mathrm{~m}$ and $d_{\text {lat }}=1000 \mathrm{~m}$. As $n$ increases the period and amplitude of the motion decrease. Hence, the relative positions of the spacecraft are nearly constant for large $n$.

If the relative motion of a formation is nearly constant over an orbit the integral can be neglected in

$$
W_{\alpha}=\frac{1}{\eta_{s} T} \int_{0}^{T} \sum_{i=1}^{n-1} \sum_{j=i+1}^{n} w\left(\alpha_{i j}\right) d t
$$


and Hir can be expressed ins 15 ir where

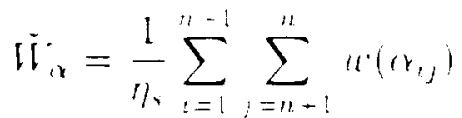

With Eqs. (23), (29), and (36) the rools are available to express $W_{\alpha}$ analytically. We are interested in the dimensions of the rotating formation that result in optimum performance. Recall that the shape of the rotating formation is determined by $e$ and $i$. The analytic expression of $W_{\alpha}$ is a non-trivial function in terms of six variables. A simplification of the expression is necessary to solve for optimum configurations in terms of $e$ and $i$. Recall that $W_{\alpha}$ is not dependent on $a$. Therefore, the analysis can be continued without concern for the specific value of semimajor axis.

To obtain an expression for $W_{\alpha}$, we need to evaluate the summation in Eq. (36) for a given $n$. A value of $n=4$ is chosen to demonstrate the techniques used to obtain the optimum. Results for several values of $n$ are presented later to determine trends in the optimum solutions for changing $n$.

Assuming $n=4$ in Eqs. (23), (29), and (36), $W_{\alpha}$ can be expressed in terms of $e, i$, $c_{1}, c_{2}$, and $c_{3}$. The results can be expanded to third-order in small $e$ and $i$ about $e=0$ and $i=0$ to obtain an approximate expression. However, the resulting expression has a singularity at $e=0$. Plotting the exact expression for $W_{\alpha}$ in negative $i$ and $e$, as shown in Figure 7, lends some understanding: there is a cusp at $e=0$ and $i=0$.

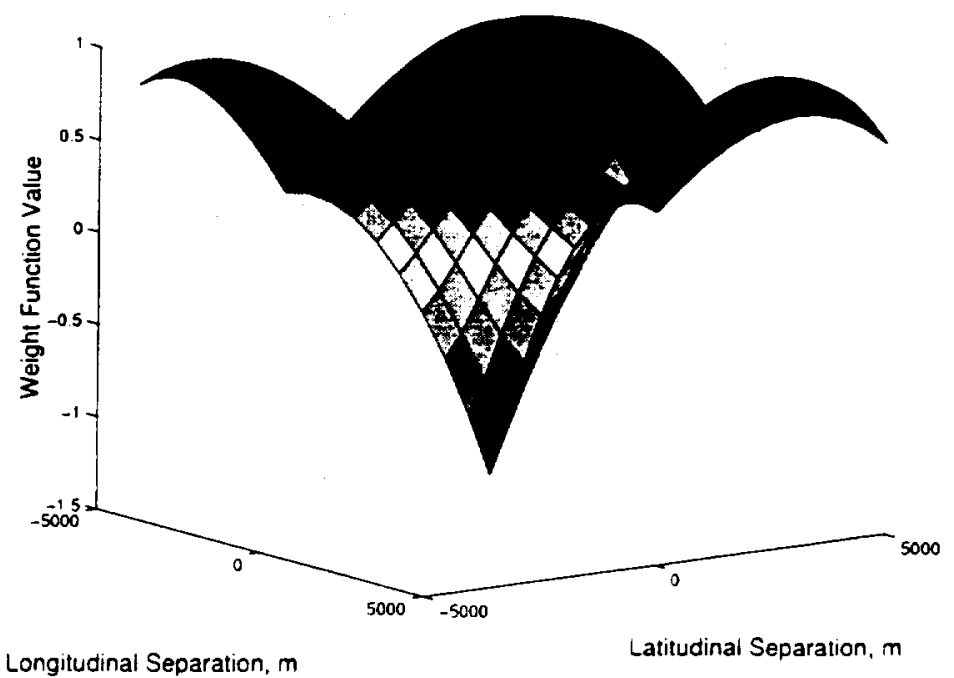

Figure 7: The Singularity in Eccentricity 
The singularity in tean be aroided by expanding about another point. To do so we make al substitution using

$$
e=\frac{i}{2}(1+\epsilon)
$$

where $0<\epsilon \ll 1$ is a small dimensionless parameter. Expanding the resulting expression to third-order in $i$ and $\epsilon$ yields an approximate expression for $W_{\alpha}$ :

$W_{a}=-\left(\frac{11 c_{2}}{18}+\frac{13 c_{2}}{18 \sqrt{2}}\right) i^{3}+\frac{8 c_{1} i^{2} \epsilon}{3}+\frac{8 c_{1} i^{2}}{3}+\frac{c_{2} \epsilon^{2} i}{6 \sqrt{2}}+\left(\frac{\sqrt{2} c_{2}+c_{2}}{3}\right) \epsilon i+\frac{2 \sqrt{2} c_{3}+2 c_{2}}{3}+c_{3}$

To solve for the $e$ and $i$ which results in an optimum solution, we must solve the set of simultaneous equations

$$
\frac{\partial W_{a}}{\partial i}=0 \quad \frac{\partial W_{a}}{\partial e}=0
$$

for $e$ and $i$. However, expressions for $\partial W_{a} / \partial i$ and $\partial W_{a} / \partial e$ in terms of $e$ must be found. Differentiating Eq. (38) with respect to $i$ yields

$$
\frac{\partial W_{a}}{\partial i}=\frac{64 c_{1}(1+\epsilon) i+c_{2}\left(8(1+\sqrt{2})+4(1+\sqrt{2}) \epsilon+\sqrt{2} \epsilon^{2}-(22+13 \sqrt{2}) i^{2}\right.}{12}
$$

To obtain an expression in terms of $e$ a substitution using

$$
\epsilon=2 \frac{e}{i}-1
$$

is performed to obtain

$$
\frac{\partial W_{a}}{\partial i}=\frac{128 c_{1} e i^{2}+c_{2}\left(4 \sqrt{2} e^{2}+4(2+\sqrt{2}) e i+i^{2}\left(4+5 \sqrt{2}-(22+13 \sqrt{2}) i^{2}\right)\right)}{12 i^{2}}
$$

Taking the partial derivative of $W_{a}$ with respect to $\epsilon$ yields

$$
\frac{\partial W_{a}}{\partial \epsilon}=\frac{i^{2}\left(c_{2}(2+2 \sqrt{2}+\sqrt{2} \epsilon)+16 c_{1} i\right)}{12}
$$

To determine an expression in terms of $e$ a multiplication by $\partial \epsilon / \partial e$ is performed where

$$
\frac{\partial \epsilon}{\partial e}=2 / i
$$

The derivative with respect to $e$ is

$$
\frac{\partial W_{a}}{\partial e}=\frac{\partial W_{a}}{\partial \epsilon} \frac{\partial \epsilon}{\partial e}=\frac{i\left(16 c_{1} i^{2}+c_{2}(2 \sqrt{2} e+(2+\sqrt{2}) i)\right)}{12}
$$

Solving Eqs. (42) and (45) simultaneously yields expressions for $e$ and $i$ that yield an optimum in $W_{\alpha}$. The expressions are complex and not shown here. However, there is a relation between the optimum $e$ and $i$ such that

$$
e_{\text {opt }}=i_{\text {opt }} / 2
$$


Recall that we assume $n=t$ in orrter to obtain an explicit expression for $\|_{a}$. Therefore, Eq. (46) appears to be dependent on the assumption that the formation is composed of four spacerraft. However, the same result is obtaimed using $n=2,3,4,5,6,8,10$.

Equation (46) has several interesting physical implications. Substituting the relations for $e$ and $i$ from Eq. (20) into Eq. (46) yields $\delta_{l o n}=\delta_{\text {lat }}$. Hence, the ideal rotating formation according to $H_{\alpha}^{\circ}$ is circular.

By substituting $e=i / 2$ into Eq. (36) an expression is obtained for $W_{\alpha}$ in terms of $n, i, c_{1}, c_{2}$, and $c_{3}$. Recall that the only assumptions made to arrive at Eq. (36) are small angles and that the integrand in Eq. (35) is constant in time. To simplify $W_{\alpha}$, we expand in a power series to second-order in $i$ and investigate a larger formation with $n=8$. A second order expansion in $i$ for $n=8$ yields

$$
W_{a}=\frac{16 c_{1}}{7} i^{2}+c_{2}\left(\frac{4}{7 \sqrt{2-\sqrt{2}}}+\frac{2 \sqrt{2}}{7}+\frac{2}{7}\right) i+c_{3}
$$

To provide a more intuitive understanding of the results, a new form for the parabolic weight function of Eq. (23) is introduced:

$$
w_{\alpha}\left(\alpha_{i j}\right)=\frac{1}{\alpha_{s}}\left(\alpha_{i j}-\alpha_{u}\right)\left(\alpha_{i j}-\alpha_{l}\right)
$$

where $\alpha_{u}$ and $\alpha_{l}$ are the upper and lower limits on angular separation respectively and $\alpha_{s}$ is a normalization factor. The constants $c_{1}, c_{2}$, and $c_{3}$ from Eq. (23) can be written in terms of $\alpha_{u}, \alpha_{l}$, and $\alpha_{s}$ using

$$
\begin{aligned}
& c_{1}=1 / \alpha_{s} \\
& c_{2}=-\left(\alpha_{u}+\alpha_{l}\right) / \alpha_{s} \\
& c_{3}=\alpha_{u} \alpha_{l} / \alpha_{s}
\end{aligned}
$$

For circular formations, the radius of the formation is simply the inclination of the orbits composing the formation. Therefore, solving for the optimum radius, $r_{o}$, from Eq. (47) and substituting for $c_{1}, c_{2}$, and $c_{3}$ using Eqs. (49-51) we obtain

$$
r_{o}=\left(\frac{1}{16}+\frac{\sqrt{2}}{16}+\frac{1}{16}(\sqrt{2-\sqrt{2}}+\sqrt{2+\sqrt{2}})\right)\left(\alpha_{l}+\alpha_{u}\right)
$$

A new angle, $\alpha_{m}$, is defined such that

$$
\alpha_{m}=\frac{\alpha_{u}+\alpha_{l}}{2}
$$

This angle is the ideal separation angle according to the instantaneous weight function $w_{\alpha}$. The optimum radius, $r_{o}$, can be rewritten in terms of $\alpha_{m}$ :

$$
r_{0}=\left(\frac{1}{8}+\frac{\sqrt{2}}{8}+\frac{1}{8}(\sqrt{2-\sqrt{2}}+\sqrt{2+\sqrt{2}})\right) \alpha_{i n}
$$


The same terhnichuts au be appled to find the optimum radius for ditferent values of 11 . The results for clitterent values of $n$ are

$$
\begin{aligned}
& n=2 \quad r_{o}=\frac{1}{2} \alpha_{m} \\
& n=3 \quad r_{o}=\left(-8 \sqrt{3} \alpha_{m}\right) /\left(-24+\sqrt{3} \alpha_{m}\right) \\
& n=4 \quad r_{o}=\frac{1}{4}(1+\sqrt{2}) \alpha_{m} \\
& n=5 \quad r_{o}=\frac{1}{5}(\sqrt{5+2 \sqrt{5}}) \alpha_{m} \\
& n=6 \quad r_{o}=\frac{1}{6}(2+\sqrt{3}) \alpha_{m} \\
& n=8 \quad r_{o}=\frac{1}{8}(1+\sqrt{2}+(\sqrt{2-\sqrt{2}}+\sqrt{2+\sqrt{2}})) \alpha_{m} \\
& n=10 \quad r_{o}=\frac{1}{10}\left(1+\sqrt{5}+\left(\sqrt{\frac{10}{4}}-\frac{\sqrt{5}}{2}+\sqrt{\frac{10}{4}}+\frac{\sqrt{5}}{2}\right)\right) \alpha_{m} \\
& n=12 \quad r_{o}=\frac{1}{12}(2+\sqrt{2}+\sqrt{3}+(\sqrt{2-\sqrt{3}}+\sqrt{2+\sqrt{3}})) \alpha_{m}
\end{aligned}
$$

There are several interesting trends seen in the equations for $r_{o}$ using different values of $n$. The equations are functions only of $\alpha_{m}$. Therefore, the optimum configuration for a given $n$ depends only on the ideal separation angle according to the instantaneous weight function $w_{\alpha}$. The shape of the parabola does not affect the results. Furthermore, with the exception of $n=3$, the optimum radii solutions are linear functions of $\alpha_{m}$.

The relation between $r_{o}$ and $n$ is not immediately obvious. However, upon close inspection we can determine a more general form of $r_{o}$ for several values of $n$. For $n=2,4,6 r_{o}$ can be expressed as

$$
r_{o}=\frac{1}{n}\left(\frac{n-2}{2}+\sqrt{\frac{n}{2}}\right) \alpha_{m}
$$

A plot of the optimum radius over a range of $n$ values is seen in Figure 8 . The points are the analytic solutions according to Eqs. (55-62) for $\alpha_{m}=0.000375 \mathrm{rad}$. The curve is a plot of the empirical solution in Eq. (63). The points for $n=2,4,6$ fall exactly on the curve. This result is expected because the empirical function is derived from the equations of $r_{o}$ for $n=2,4,6$. The values of $r_{0}$ for $n=3,5$ are in good agreement with the empirical solution. However, for $n>8$ the accuracy of the empirical function degrades. The empirical function predicts a downward trend in $r_{o}$ for $n>8$. However, the analytic solution appears to approach a limiting value.

The limit of $r_{o}$ as $n$ approaches infinity can be determined using simple geometric relations. For $n \rightarrow \infty, w_{p}\left(\alpha_{i j}\right)$ is the same for all $i$ where $i \neq j$. Therefore, we only 


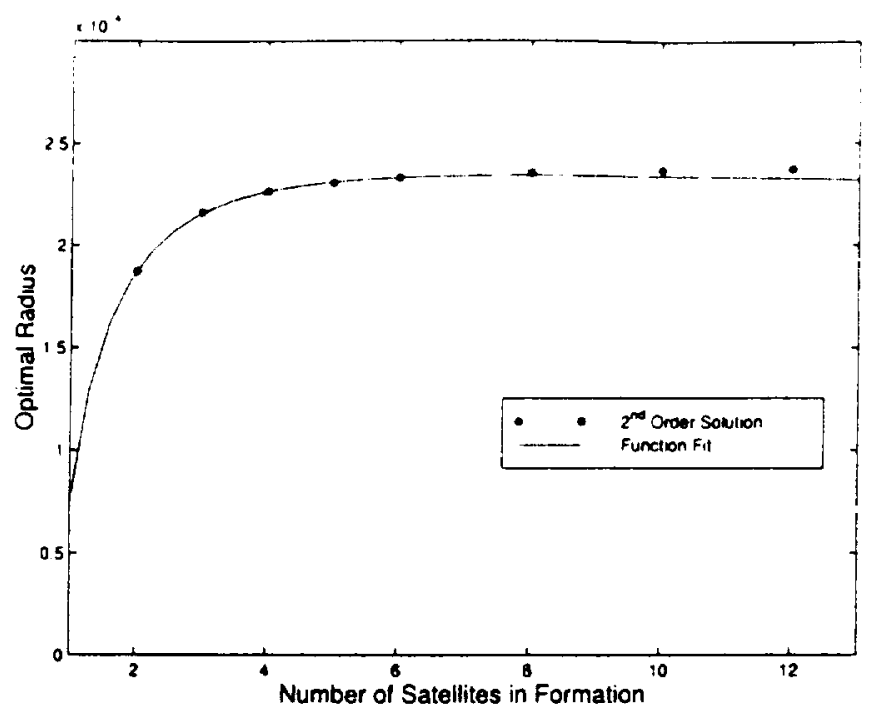

Figure 8: Optimum Radius for the Rotating Formation

need to evaluate $w_{p}\left(\alpha_{i j}\right)$ for an arbitrary $i$. Thus for $n \rightarrow \infty$

$$
\tilde{W}_{\infty}=\lim _{n \rightarrow \infty} \frac{1}{\eta_{s}} \sum_{i=1}^{n-1} \sum_{j=n+1}^{n} w_{p}\left(\alpha_{i j}\right)=\lim _{n \rightarrow \infty} \frac{1}{n-1} \sum_{j=2}^{n} w_{p}\left(\alpha_{j}\right)
$$

Figure 9 shows two arbitrary radius vectors, $\mathbf{r}_{1}$ and $\mathbf{r}_{2}$, defining two spacecraft positions in a circular formation. Angle $r_{f}$ is the angular radius of the formation. The angle around the circle between $\mathbf{r}_{1}$ and $\mathbf{r}_{2}$ is denoted $\theta$. Using these definitions Eq. (64) can be written as

$$
\tilde{W}_{\infty}=\lim _{n \rightarrow \infty} \frac{1}{n-1} \sum_{j=2}^{n} w_{p}\left(\alpha_{j}\right)=\frac{1}{2 \pi} \int_{0}^{2 \pi} w_{p}(\alpha) d \theta
$$

From inspection of Figure 9 we can write

$$
\begin{gathered}
\mathbf{r}_{1}=\left[\begin{array}{lll}
\sin r_{f} & 0 & \cos r_{f}
\end{array}\right]^{T} \\
\mathbf{r}_{2}=\left[\begin{array}{lll}
\sin r_{f} \cos \theta & \sin r_{f} \sin \theta & \cos r_{f}
\end{array}\right]^{T}
\end{gathered}
$$

Recall that $W_{\alpha}$ is not dependent on the semimajor axis. Therefore, we use unit vector representations of the spacecraft positions without loss of generality. L'sing $r_{1}=r_{2}=1, \alpha$ can be expressed as

$$
\alpha=\left\|\mathbf{r}_{1} \times \mathbf{r}_{2}\right\|
$$

for small $\alpha$. Substituting Eqs. (66-67) into Eq. (68) we obtain

$$
\alpha=\sqrt{\sin ^{2} r_{f}\left(-2 \cos ^{2} r_{f}(-1+\cos \theta)+\sin ^{2} r_{f} \sin ^{2} \theta\right)}
$$




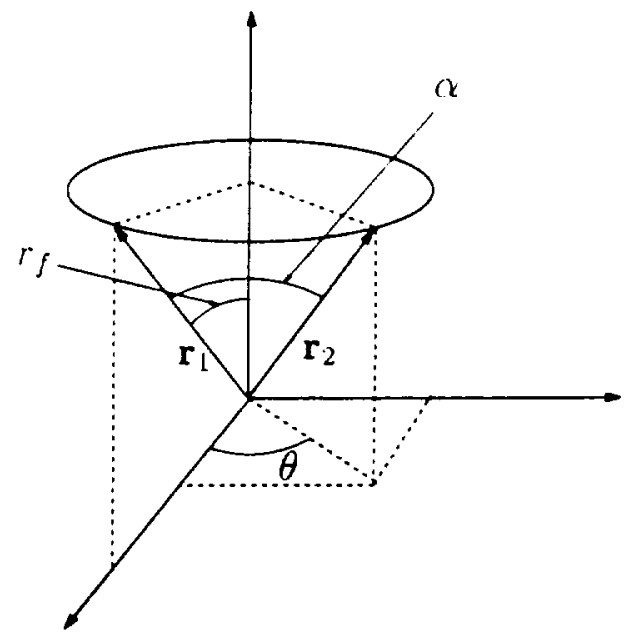

Figure 9: Circular Formation Geometry for $n=\infty$

Substituting Eqs. (69) and (53) into Eq. (48) the integral in Eq. (65) can be evaluated:

$$
\begin{aligned}
\tilde{W}_{\infty}= & \frac{1}{16 \pi \alpha_{s}}\left(-7 \pi-16 \pi \alpha_{l} \alpha_{u}+3 \pi \cos 4 r_{f}+8 \alpha_{m}\left(k_{2}-k_{1}\right)\right) \\
& +\frac{1}{16 \pi \alpha_{s}}\left(4 \cos 2 r_{f}\left(\pi+2 \alpha_{m}\left(k_{2}-k_{1}\right)\right)+32 \alpha_{m} \sqrt{\sin ^{2} r_{f}}\right)
\end{aligned}
$$

where $k_{1}$ and $k_{2}$ are given by

$$
\begin{aligned}
& k_{1}=\ln \left[1-\sqrt{\sin ^{2} r_{f}}\right] \\
& k_{2}=\ln \left[1+\sqrt{\sin ^{2} r_{f}}\right]
\end{aligned}
$$

To solve for the optimum radius as $n \rightarrow \infty$ we need to solve

$$
\frac{\partial \tilde{W}_{\infty}}{\partial r_{f}}=0
$$

However, the resulting derivative is a transcendental function in terms of $\alpha_{m}$ and $r_{f}$ and cannot be explicitly solved for the optimum radius. Therefore, Eq. (70) is expanded in small $r_{f}$ to second order:

$$
\tilde{W}_{\infty} \approx \frac{1}{\alpha_{s}}\left(2 r_{f}^{2}-\frac{4 \alpha_{m} r_{f}}{\pi}+\alpha_{l} \alpha_{u}\right)
$$

Defining $r_{\infty}$ as the optimum radius as $n \rightarrow \infty$ and solving Eq. (74) for $r_{\infty}$ obtains

$$
r_{\infty}=\frac{2}{\pi} \alpha_{m}
$$




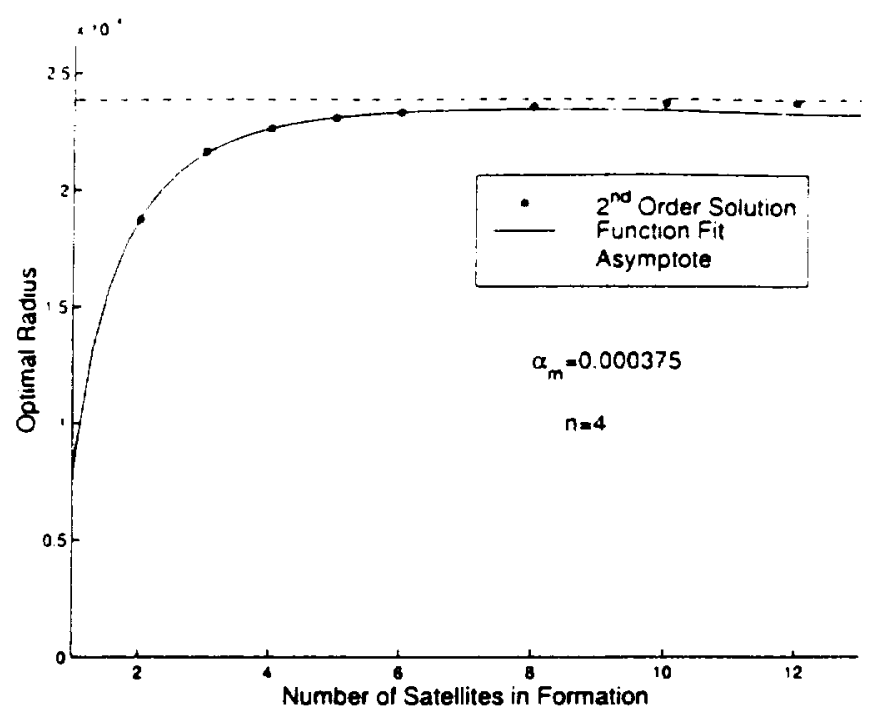

Figure 10: Optimum Radius with Asymptote vs. $n$

Like $r_{o}$ for small $n, r_{\infty}$ is only a function of $\alpha_{m}$. Therefore, the shape of the parabolic instantaneous weight function does not affect the optimum solution for large $n$. In Figure 10, the solution of $r_{o}$ for a range of $n$ values is plotted with the asymptote $r_{\infty}$. The approximation for the asymptote is in good agreement with the analytic solution for $r_{o}$ for large $n$.

Recall that several simplifying assumptions are made to obtain an analytic solution for $W_{\alpha}$ and the limiting value as $n \rightarrow \infty$. We need to determine if the simplifications yield solutions that are in good agreement with the exact solutions. Plots of $W_{\alpha}$ for $n=3,4,8$, and 12 are shown in Figures 11-12 for a range of $\alpha_{m}$. The data points are the exact numerical solutions for different radii obtained using a Runge-Kutta fourth order integrator to solve Eq.(21). The curves are the approximate analytic solutions for $W_{\alpha}$. The approximate solutions are in excellent agreement with the exact solution for low $\alpha_{m}$. For high $\alpha_{m}$, on the order of $\alpha_{m}=0.25$, accuracy of the approximate solution for $W_{\alpha}$ is poor. A decrease in accuracy is expected for high $\alpha_{m}$ because the small angle approximation was used in the development of the approximate solution. However, the optimum in performance occurs at nearly the same point according to both the exact and approximate solutions even for $\alpha_{m}=0.25$. In Figure 13, plots of the exact $v s$. approximate optima are shown for a range of $\alpha_{m}$. The approximate solutions are accurate even for large $\alpha_{m}$ on the order of $\alpha_{m}=0.5$. In the range of $0.5<\alpha_{m}<1$ the accuracy of the approximate solution degrades. However, the approximate results are still useful in this range. We suggest that for $0.5<\alpha_{m}<1$ the approximate solutions be used as an initial guess to solve for the exact optimum using numerical techniques. 

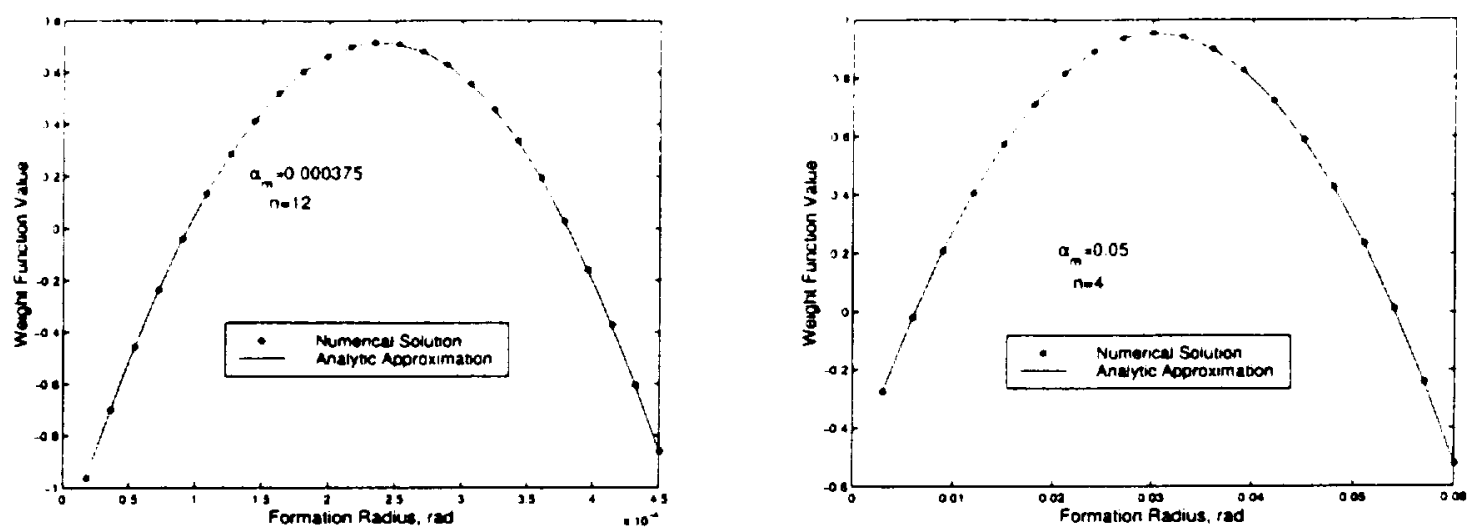

Figure 11: Approximate and Exact Solutions for $W_{\alpha}$
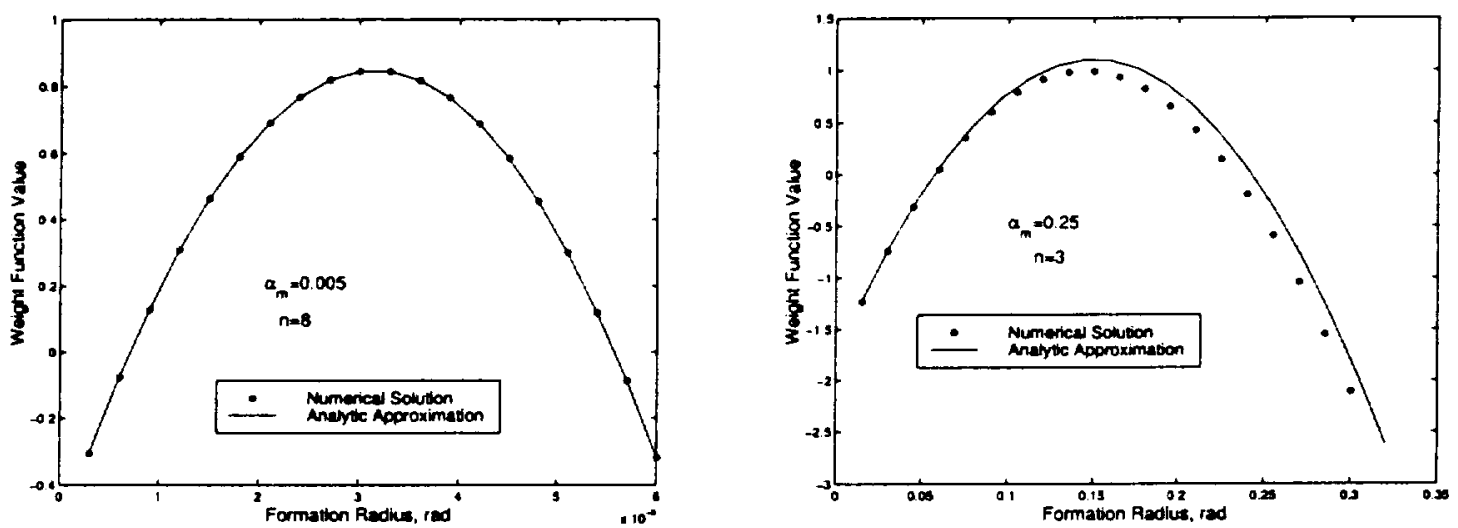

Figure 12: Approximate and Exact Solutions for $W_{\alpha}$
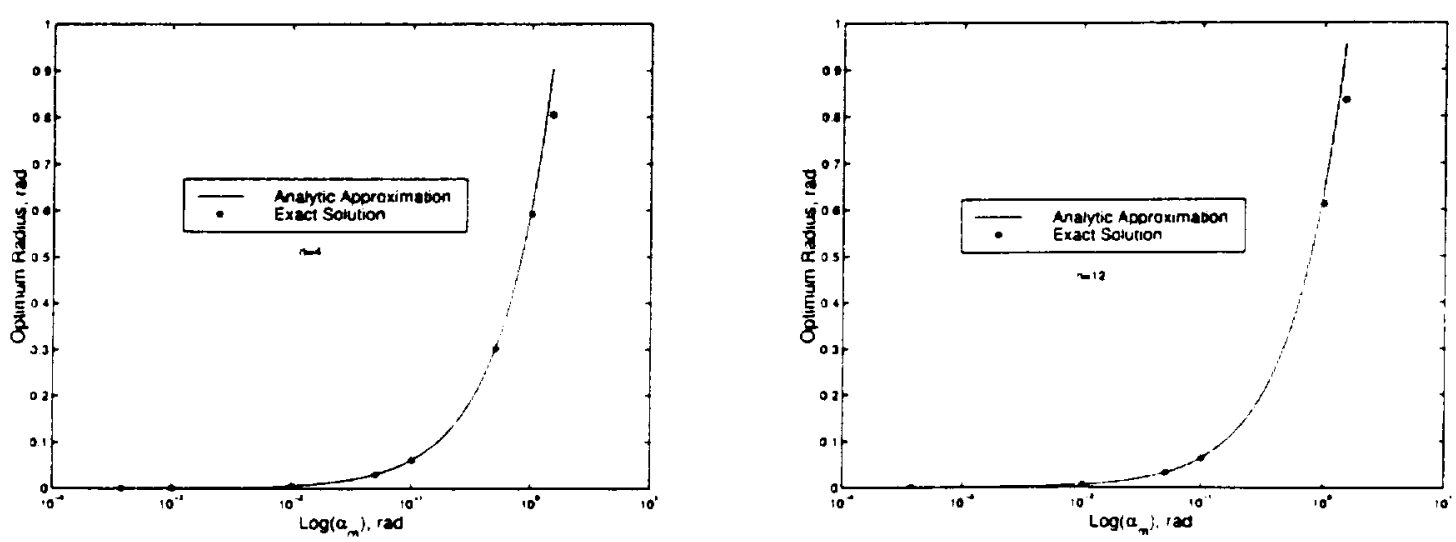

Figure 13: Approximate and Exact Optima vs. $\alpha_{m}$ 


\section{CONCLUSIONS}

In this paper an algorithm is developed to place $n$ satellites equally spaced in time around an ellipse in the relative reference frame in terms of the classical orbital elements. The dimensions of the ellipse-like path are shown to be simple functions of orbit eccentricity and inclination. The performance of the formation is investigated using a performance metric based on the orbit averaged sum of the angular separations between spacecraft. The exact expression is a non-trivial function of orbit eccentricity, inclination, the number of spacecraft, and the constants that define the instantaneous weight function. However, several simplifying assumptions allow for an analytic approximation. Lsing the resulting approximate equation for $W_{\alpha}$, optimal configurations are found for different values of $n$. We show that, according to the angular performance measure, the optimal rotating formation is circular. The optimal radii for different values of $n$ are found to be simple functions of $\alpha_{m}$, the ideal separation angle according to the instantaneous weight function. Interestingly, the optimal configuration does not depend on the shape of the parabola chosen for the instantaneous separation metric. For $n<8$, the optimal radius is $r_{o}=((n-2) / 2+\sqrt{n / 2}) \alpha_{m} / n$, whereas for large $n(>8)$, the asymptotic limit $r_{o} \rightarrow 2 \alpha_{m} / \pi$ is quite accurate.

\section{ACKNOWLEDGMENTS}

The first author was supported by a grant from the Guidance Navigation and Control Center at NASA Goddard Space Flight Center, under the direction of David Folta and David Wiedow. The second author was supported by a grant from the Air Force Office of Scientific Research, under the direction of Arje Nachman.

\section{NOTATION}

$\begin{array}{ll}a & \text { semimajor axis, m } \\ c_{1}, c_{2}, c_{3} & \text { constants defining parabolic weight } \\ d & \text { spatial separation between two spacecraft, } \mathrm{m} \\ e & \text { orbit eccentricity } \\ \mathcal{F}_{I} & \text { inertial reference frame } \\ \mathcal{F}_{\text {rel }} & \text { relative reference frame } \\ \mathcal{F}_{R} & \text { rotating reference frame } \\ \mathcal{F}_{p} & \text { perifocal reference frame } \\ i & \text { orbit inclination, rad } \\ \ell & \text { true longitude at epoch, rad } \\ n & \text { number of spacecraft in formation } \\ n_{s} & \text { number of distinct angles in a formation } \\ r_{\infty} & \text { optimum radius as } n \rightarrow \infty, \text { rad } \\ r_{0} & \text { optimum radius for rotating formation. rad }\end{array}$




\begin{tabular}{|c|c|}
\hline$T$ & orbital period. s \\
\hline$t_{6}$ & 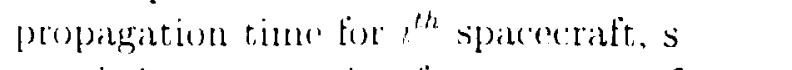 \\
\hline$t_{s}$ & "platl time separation for $n$ spalcecraft, $s$ \\
\hline$H_{c r}$ & orbit performance matisure \\
\hline$W_{\infty}$ & $I_{c}$ is $n \rightarrow \infty$ \\
\hline$W_{a}$ & approximate expression for $W_{\alpha}$ \\
\hline$w_{\alpha}$ & instantaneous position metric \\
\hline$\alpha_{i j}$ & angle between the $i^{\text {th }}$ and $j^{\text {th }}$ satellites, rad \\
\hline$\alpha_{l}$ & lower limit on angular separation, rad \\
\hline$\alpha_{m}$ & ideal angular separation, rad \\
\hline$\alpha_{s}$ & scaling factor, rad \\
\hline$\alpha_{u}$ & upper limit on angular separation, rad \\
\hline$\delta_{l o n}$ & formation longitudinal separation, rad \\
\hline$\delta_{\text {lat }}$ & formation latitudinal separation,rad \\
\hline$\eta$ & orbit mean motion, rad $/ \mathrm{s}$ \\
\hline$\nu$ & true anomaly, rad \\
\hline$\Omega$ & longitude of ascending node, rad \\
\hline$\omega$ & argument of periapsis, rad \\
\hline
\end{tabular}

\section{REFERENCES}

[1] Folta, D. C., Bordi, F., and Scolese, C., "Field of View Location for Formation Flying Polar Orbiting Missions," Advances in Astronautical Sciences, Vol. 75, No. 2, 1991, pp. 949-965.

[2] Folta, D. C., Bordi, F., and Scolese, C., "Considerations on Formation Flying Separations for Earth Observing Satellite Missions," Advances in Astronautical Sciences, Vol. 79, No. 2, 1992, pp. 803-822.

[3] Folta, D. C., Newman, L. K., and Gardner, T., "Foundations of Formation Flying for Mission to Planet Earth and New Millennium," In Astrodynamics Conference Proceedings, 1996, pp. 656-666.

[4] Decou, A. B., "Orbital Station-Keeping for .Multiple Spacecraft Interferometry." Journal of the Astronautical Sciences, Vol. 39, No. 3. 1991, pp. 283-297.

[5] Gramling, C. J., Lee, T., Niklewski, D. J., and Long, A. C., "Relative Navigation for Autonomous Formation Flying Spacecraft," Advances in Astronautical Sciences, Vol. 97. No. 1, 1998, pp. 405-431.

[6] Hartman, K. R., Gramling, C. J., Lee, T., Kelbel. D. A., and Long. A. C., "Relative Vavigation for Spacecraft Formation Flying." Advances in Astronautical Sciences, Vol. 100, Nio. 2. 1998, pp. 685-699. 
[T] Cunim. J. R. and Buain. R. J., "Spacecraft Autonomous Vavigation tor Fomation

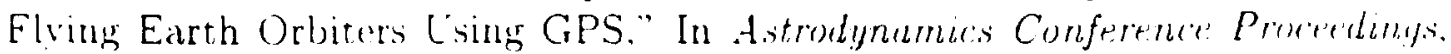
1996, pp. $72 \cdot 2-732$.

[8] Arlams, J., Robertson, A., Zimmerman, K., and How, J.. "Technologies for Sparecraft Formation Flying," In Proceedings of the Institute of Navigation GPS, 1096. pp. $1321-1330$.

[9] How, J. P., Twiggs, R., Weidow, D., Hartman, K., and Bauer, F., "Orion: A LowCost Demonstration of Formation Flying in Space Using GPS," In Astrodynamics Conference Proceedings, 1998, pp. 276-286.

[10] Battin, R. H., An Introduction to the Mathematics and Methods of Astrodynamics, Amercian Institute of Aeronautics and Astronautics, Reston, 1987.

[11] Chichka, D. F., "Dynamics of Clustered Satellites via Orbital Elements," In AAS/AIAA Astrodynamics Specialist Conference Proceedings, 1999, pp. 969988.

[12] Hughes, S. P. and Hall, C. D., "Formation Flying Performance Measures for Earth Pointing Missions," In Flight Mechanics Symposium, 1999, pp. 309-318. 